\title{
Notas para una revisión crítica del concepto de "poder"*
}

\section{Alberto Montbrun**}

\begin{abstract}
Resumen: El concepto de poder ha sido uno de los más tradicionales y arraigados de la ciencia política, desde la temprana reflexión de los griegos hasta la actualidad. Es aún hoy un término de extraordinaria vigencia en el lenguaje científico y popular. Sin embargo, cuando indagamos en su real materialidad, advertimos que el mismo es elusivo, confuso y difuso. Desde la emergencia del positivismo, pasando por las revoluciones burguesas, el concepto se mantuvo más o menos inalterado. Con la emergencia del marxismo, de las ideologías modernas y de las escuelas críticas se persiguió su racionalización y redireccionamiento, sin cuestionar la esencia de su significado. Sugerimos que a partir de la Teoría de la Complejidad y de la Teoría de Redes, el concepto podría muy bien dejarse fuera de la ciencia política o quedar limitado sólo a los alcances de la fuerza o coacción.
\end{abstract}

Palabras clave: poder, sistemas adaptativos complejos, teoría de redes

\section{Notes for a critical review on the concept of "power"}

\begin{abstract}
The concept of power has been one of the most traditional and rooted in political science, since the early considerations of the Greeks up to the present. It is still today a term of extraordinary actuality in popular and scientific language. However, when we inquire into its real materiality, we note that it is elusive, confuse and diffuse. Since the emergence of positivism, through the bourgeois revolutions, the concept remained more or less unchanged. With the emergence of Marxism, of modern ideologies and critical schools, its rationalization and redirection was persecuted, but without questioning the essence of its meaning. We suggest that, starting from the Complexity Theory and Network Theory, that the concept could well be left out of political science or be confined only to the scope of force or coercion.
\end{abstract}

Key words: power, complex adaptive systems, network theory

\section{Notas para uma análise crítica do conceito de "poder"}

Resumo: O conceito de poder tem sido uma das mais tradicionais e enraizados na ciência política a partir da reflexão inicial desde os gregos até o presente. É ainda uma noção de força extraordinária em linguagem popular e cien-

\footnotetext{
* El presente paper es parte de la investigación sobre "Bases educativas y lineamientos para la transformación de la Policía en un modelo de organización inteligente”, SECYT - Universidad Nacional de Cuyo, 2007-2009 y 2009-2011. El equipo de investigación está conformado por Alberto Montbrun (Director), Edgardo Valenzuela, Paula Maurano, Francisco Immerso, Maximiliano Legrand, Liliana Porras y Miguel Mansilla.

** Universidad Nacional de Cuyo, Mendoza, Argentina. Email: albertomontbrun@yahoo.com
} 
tífico. No entanto, quando indagamos sobre sua materialidade real, podemos constatar que ele é evasivo, vago e confuso. Desde o surgimento do positivismo, por meio das revoluções burguesas, o conceito foi mais ou menos inalterado. Com o surgimento do marxismo, das ideologias modernas e escolas críticas foram perseguidos seu racionalização e redirecionamento, sem questionar a essência do seu significado. Sugerimos que, a partir da Teoria da Complexidade e Teoria da redes, o conceito poderia muito bem ser deixado de fora da ciência política ou ser confinada ao âmbito de aplicação da força ou coação.

poder

Palavras-chave: poder, sistemas adaptativos complexos, a teoria da rede:

Recibido: 15.01.2010

Aceptado: 07.02.2010

$* * *$

\section{Introducción}

Existen innumerables situaciones sociales en las cuales algunas personas determinan y condicionan las conductas de otras. Aún más, podemos seguramente afirmar que existen múltiples situaciones sociales en las que unas personas imponen determinadas conductas a otras, aún contra la voluntad de estas. Esto ha hecho que el concepto de “poder" haya recibido a lo largo de los siglos múltiples definiciones y significados. No obstante ello, lo encontramos en general ligado a conceptos como los de "dominación”, “coacción”, “mando y obediencia”, “imposición”, “amenaza”, “fuerza” y tantos otros.

Es nuestra inquietud indagar qué pueden aportar las nuevas vertientes científicas englobadas genéricamente bajo la denominación de "nuevo paradigma científico” o “paradigma científico autoorganizativo” al estudio de este concepto a fin de determinar si el mismo mantiene su vigencia e importancia como elemento central del análisis de la política.

Para ello reseñaremos brevemente las nociones de poder en la doctrina política tradicional y formularemos luego algunas breves referencias al manejo del concepto en el contexto de las revoluciones burguesas. Finalmente encararemos los aportes de los estudios sobre sistemas adaptativos complejos (SAC) y teoría de redes a fin de evaluar su pertinencia en la clarificación del concepto. Sólo esbozaremos al final algunas conclusiones provisorias sobre este debate, sin ánimo de fijar posiciones definitivas en un tema siempre abierto a los aportes enriquecedores.

\section{El concepto de poder en la doctrina política}

En su acepción tal vez más básica y elemental, el concepto de poder aparece en la doctrina política ligado siempre a la capacidad de unas personas de imponer determinadas conductas a otras personas, aún contra la vo- 
luntad de éstas. El Licenciado Walter Cueto, profesor de la Facultad de Ciencias Políticas de la UNC, enumera las siguientes definiciones y sus respectivos autores ${ }^{1}$ :

Robert Dahl² : En el nivel mas general, los términos de poder de la ciencia social moderna se refieren a subconjuntos de relaciones entre unidades sociales tales que los comportamientos de una o más unidades sociales (las unidades que obedecen R) depende en cualquier circunstancia del comportamiento de otras unidades sociales (las unidades que controlan, C).

David Easton $^{3}$ : El poder es un fenómeno de relaciones, no es una cosa que alguien posea. El poder es una relación en la cual una persona o grupo puede determinar las acciones de otro, en forma tal que satisfaga los fines del primero.

Carl Friedrich ${ }^{4}$ : El poder se presenta como una relación interpersonal que se manifiesta con la obediencia, o sea con un comportamiento que indica adecuadamente que A, B y C hacen lo que L desea.

Raymond Aron $^{5}$ : El poder es la capacidad de un individuo para determinar la conducta de otros. En su sentido más general, el poder es la capacidad de hacer, producir o destruir.

Harold Laswell y Abraham Kaplan ${ }^{6}$ : Poder es la participación en la toma de decisiones. La adopción de decisiones constituye un proceso interpersonal. El poder representa una relación interpersonal.

Richard Schermerhorn ${ }^{7}$ : Podemos definir el poder como la relación procesal entre dos partícipes modalmente caracterizados por:

a. la influencia asimétrica, en la cual una perceptible probabilidad de decisión depende de uno de los dos partícipes, incluso a pesar de la resistencia del otro; y

b. por el predominio de las sanciones negativas, reales en cuanto amenaza, como característica de la conducta partícipe dominante.

Amitai Etzioni ${ }^{8}$ : El poder es la capacidad de superar toda resisten-

\footnotetext{
${ }^{1}$ En un paper de la Facultad de Ciencias Políticas de la Universidad Nacional de Cuyo. Muchas definiciones pueden verse en Labourdette, Sergio; El poder. Hacia una teoría sistemática; Ed. Universidad de Belgrano, Buenos Aires, 1984. También en Oro Tapia, Luis; ¿Qué es la Política?; Ril Editores, Chile, 2003.

${ }^{2}$ Dahl, Robert; The Concept of Power; Rev. Behavioral Science, Vol. II, 1957

${ }^{3}$ Easton, David; Política Moderna; Ed. Letras, México, 1968. p. 149

${ }^{4}$ Friedrich, Carl; El hombre y el Gobierno; 1968, p. 182

${ }^{5}$ Aron, Raymond; Democracia y totalitarismo; Editorial Seix Barral, Barcelona, 1968

${ }^{6}$ Laswell y Kaplan; Power and Society, Yale University Press, 1950, p. 75

${ }^{7}$ Schermerhorn, Richard; El poder y la sociedad; PAIDOS, Buenos Aires, 1968

${ }^{8}$ Etzioni, Amitai; La sociedad activa. Una teoría de los procesos sociales y políticos; Aguilar, Madrid, 1978.
} 
cia o parte de ella para introducir cambios a pesar de la oposición.

Eduard Spranger ${ }^{9}:$ El poder es la capacidad, y también en la mayoría de los casos, la voluntad de imponer a los demás las propias orientaciones valorativas con motivo permanente o pasajero.

Dentro de la misma tradición, Julien Freund expresa que el poder es el mando estructurado socialmente, dividido en funciones jerárquicas y llevado por una o varias capas sociales variables según los regímenes. Señala que "ocurre con el mando político como con cualquier tipo de mando: consiste en la relación jerárquica que se establece en el seno de un grupo por la potencia que una voluntad particular ejerce sobre las otras voluntades particulares, modelando así la cohesión del grupo.

Desde una perspectiva un poco más amplia Karl Deutsch señala que el poder es la capacidad para hacer que sucedan cosas que de otro modo no habrían sucedido. Es la producción de un cambio en la distribución de probabilidades de los acontecimientos del mundo y, dado que el mundo ya está cambiando, el poder se refiere al cambio del cambio. El poder es la capacidad para alterar los cambios que ya están en proceso y que seguirán adelante sin nuestra intervención.

Para Artemio Melo, en una definición que combina los aportes de varios autores ${ }^{10}$, el poder es la energía que impulsa a la acción política, a la realización de algún valor mediante el establecimiento de un orden en una comunidad concreta a fin de asegurar el bien común.

En general hay coincidencia en los autores en torno a que las relaciones de poder tienen como características claves:

a. La de ser una relación social o una relación entre personas, es decir que el poder es siempre un fenómeno relacional.

b. La relación de poder es asimétrica, es decir hay una persona que manda y una que obedece y en tal sentido la relación tiene una fuerte unidireccionalidad.

También hay coincidencia en los autores clásicos en señalar que en la vida social el poder ha sido visto como la posibilidad de imponer la voluntad propia a los demás a través de algún medio específico: el conocimiento, la inteligencia, la fuerza, la riqueza, el dogma o cualquier factor que sirva para impulsar o constreñir otros a hacer lo que en otras circunstancia no harían.

Así mismo se reconoce en general que la obediencia tiene matices

\footnotetext{
${ }^{9}$ Spranger, Eduard; Formas de Vida; Ed. Revista de Occidente, Madrid, 1966

${ }^{10}$ Melo, Leopoldo; Compendio de Ciencia Política, Depalma, Buenos Aires, 1979
} 
que van desde las respuestas basadas en la convicción de la legitimidad del mandato o de la conveniencia en consentirlo hasta la percepción de una amenaza en caso de desobediencia o directamente el puro acatamiento forzado.

Considerando que invariablemente las definiciones de poder se mezclan o relacionan con otros conceptos como fuerza o dominio, algunos autores propusieron una más desagregada distinción de los mismos.

Max Weber propone diferenciar poder de "dominación” o "autoridad"11. Para este autor, el poder es la probabilidad de imponer la propia voluntad dentro de una relación social aún contra toda resistencia y cualquiera sea el fundamento de su probabilidad. El concepto de poder es sociológicamente amorfo. Todas las cualidades imaginables de un hombre y toda suerte de constelaciones pueden colocar a alguien en la posición de imponer su voluntad en una situación dada.

Pero incorpora como variable central de la distinción el concepto de legitimidad ${ }^{12}$ entendida genéricamente como la coherencia entre las decisiones de poder y el sistema de valores de los que deben obedecerlas. De allí se extrae la conclusión de que la dominación o autoridad es poder más legitimidad y en el caso opuesto es necesaria una mayor aplicación de la fuerza para imponer la decisión. La teoría de Weber ha tenido un fuerte arraigo en la doctrina política contemporánea.

Sin embargo, una definición nos llama la atención. Max Weber dice que el poder es “...la probabilidad de imponer la propia voluntad, dentro de una relación social, aún contra toda resistencia y cualquiera que sea el fundamento de esa probabilidad”.

Si la probabilidad es la posibilidad o factibilidad de que un acontecimiento suceda, la definición de Weber no parece haber perdido del todo vigencia en el marco de la teoría de la ciencia de la complejidad. En el contexto de la sociedad industrial de finales del siglo XIX y principios del $\mathrm{XX}$, la probabilidad a la cual hace referencia Weber seguramente era mayor que en la infinitamente más compleja sociedad de principios del siglo XXI, pero, probabilidad al fin.

A pesar de analizar el fenómeno de la dominación ${ }^{13}$, la idea de Weber acerca del poder parece acentuar una visión unidireccional del tipo "mando - obediencia” y soslaya el fenómeno interaccional pluridireccional, que, según veremos, es propio de la red.

\footnotetext{
${ }^{11}$ Weber, Max; Economía y Sociedad, Ed. FCE, México, 1993. p. 43

${ }^{12}$ Labourdette, Sergio; op. cit; p. 64 y ss.

${ }^{13} \mathrm{El}$ cual define como la probabilidad de encontrar obediencia a un mandato determinado contenido entre personas dadas.
} 
Hannah Arendt, por su parte, señala que es cuestionable que la ciencia política haya perdido la capacidad para distinguir los conceptos de poder, autoridad y fuerza al extremo de que aparecen como sinónimos en nuestros días. Para esta autora el poder es la capacidad humana de actuar concertadamente y en tal sentido es propio de toda la comunidad. La "autoridad" es el poder que ejercen unos pocos con el reconocimiento de aquellos a quienes se les pide obedecer y que no necesita del miedo ni de la coerción. La fuerza o violencia se utilizan cuando la autoridad fracasa ${ }^{14}$.

A diferencia de lo afirmado por Weber, Hannah Arendt, considera que, en sentido estricto, el poder sólo puede ser realmente efectivo, si incluye el consentimiento de los gobernados. Para Arendt, la sobrevivencia del poder está estrechamente ligada al grado de adhesión que logre suscitar y mantener en la ciudadanía. Mientras que Weber sostiene que el poder está referido siempre a la intencionalidad y a la voluntad del individuo que lo ejerce, Arendt responde que "el poder no es nunca una propiedad individual. El poder pertenece al grupo y sobrevive sólo en la medida en que el grupo permanece. Cuando decimos de alguien que se encuentra en el poder, lo que queremos decir es que su investidura de poder proviene de un cierto número de personas que lo autorizan a actuar en su nombre”.

Si desaparece el sostén y el apoyo de la colectividad o del grupo, el poder termina por desvanecerse. Para Arendt, por consiguiente, la tiranía representa así el grado supremo de la violencia y el grado mínimo de poder. De ahí su aforismo paradójico según el cual, “La forma extrema de poder es todos contra uno y la forma extrema de violencia es uno contra todos”.

Michael Foucault, en cambio, intenta otra aproximación. En vez de preocuparse por qué es el poder sugiere que habría que preguntarse más bien cómo se ejerce el poder, mediante qué tecnologías y mediante qué procedimientos se ejerce ese poder y qué consecuencias y efectos se derivan de ello. En definitiva, el poder no es una institución, no es una estructura ni una fuerza de la que dispondrían algunos: es el nombre que se le da a una situación estratégica compleja en una sociedad dada ${ }^{15}$.

En un segundo momento de su reflexión, Foucault busca precisar aún más los rasgos definitorios de las prácticas del poder. En ese esfuerzo subraya que el poder no es en modo alguno acción directa o inmediata sobre los otros. Su formulación es más compleja: el poder “actúa sobre sus acciones; una acción sobre la acción, sobre las acciones eventuales o actuales, presentes o futuras”.

\footnotetext{
${ }^{14}$ Arendt, Hannah; La condición humana; Barcelona, Paidós, 1993; Rivera Garcia, Antonio; Sobre el concepto político de "autoridad" en Hanna Arendt; Rev. Daimon, 26-2002; Avila Fuenmayor, Francisco, Algunas Ideas del Pensamiento Político de Hanna Arendt. Su impacto actual; Revista de Cencias Sociales, Vol 11, Maracaibo, 2005

${ }^{15}$ Foucault, Michel: La volonté de savoir, Gallimard, París, 1976; Un diálogo sobre el poder, Alianza Editorial, Madrid, 1981; “El sujeto y el poder”, en Revista Mexicana de Sociología, No. 3, 1988.
} 
La distinción que Foucault hace entre violencia y poder se funda precisamente en esa diferencia: mientras que la violencia se realiza sobre las cosas o sobre los cuerpos para destruir o someter, el poder supone el reconocimiento del otro como alguien que actúa o que es capaz de actuar. En ese sentido, gobernar es incidir sobre el campo de acción real o posible de los otros. De ahí la célebre reiteración de Foucault según la cual, al final de cuentas ejercer el poder no es más que "conducir conductas", valga decir: la posibilidad de ampliar o de restringir el campo de acción de los otros. De esos otros a quienes se reconoce como actuantes y responsables: como capaces de actuar y, sobre todo, de responder.

\section{El concepto de poder y las revoluciones burguesas}

Si bien las definiciones de poder que hemos reseñado son en su mayoría contemporáneas, deseamos señalar un dato que nos parece pertinente: ellas responden a una idea de poder fuertemente anclada en el reduccionismo cartesiano newtoniano, cuya base paradigmática influyó notoriamente en los pensadores políticos que definieron el perfil del Estado en la modernidad.

Para esta concepción científica, el poder es el equivalente a la "fuerza” en el campo de la física, entendida como un quantum de energía aplicado a una cosa para que haga o deje de hacer algo. Este concepto estrictamente mecánico del poder como fuerza pasó a las nacientes ciencias sociales de la mano del positivismo científico.

Tomas Hobbes, con su Leviatán (1650), John Locke, con su Segundo Ensayo sobre el Gobierno Civil (1690), Charles de Montesquieu con El espíritu de las leyes (1748) y Jean Jaques Rousseau con su teoría del Contrato Social (1762) definieron el andamiaje ideológico con el cual la burguesía encaró una nueva configuración del poder político, que se integró también con la concepción mecanicista de gobierno tripartito que consagró la Constitución de Estados Unidos de 1787. Para Hobbes, dentro de esta tradición, el poder consiste en los medios para obtener alguna ventaja. John Locke en su Segundo Tratado sobre Gobierno Civil destaca que "El fundamento del poder civil es el consenso por estar dirigido en el interés de los gobernados" y que el poder legítimo solo puede surgir de la voluntad libremente expresada de los miembros de la comunidad.

En esos años quedan definidas también las bases de la ciencia económica moderna con La riqueza de las naciones de Adam Smith (1776) y las bases del derecho penal liberal con la aparición de Los delitos y las penas del Marqués de Beccaria (1762).

Las principales características del modelo ideológico de la época son, entre otros, la consideración del individuo como el protagonista de la historia y la conceptualización del egoísmo y la competencia como inherentes a la naturaleza humana y motores del progreso. También la conside- 
ración del universo como un mecanismo con leyes inmutables y eternas y la idea de que sólo la razón nutriendo la observación metódica de la realidad permite fundar o descubrir verdades absolutas y objetivas. La economía también está sometido a leyes naturales y por lo tanto se espera por parte del poder político un laissez faire que no interfiera con las mismas y el poder político deviene de la comunidad (Nación) que se organiza a través de un pacto social y se formaliza en una constitución escrita y rígida que es a la vez un freno al poder, en tanto es una carta de garantía de los derechos individuales y además organiza la administración del poder tripartito. La teoría de la representación política, sintetizada por Sieyes, es la forma de encauzar la soberanía popular a través de un acto de delegación del poder bajo la forma de un mandato libre.

Resulta interesante destacar que las revoluciones burguesas no alteraron el concepto de poder entendido como capacidad de imponer decisiones y conductas a los súbditos, sino que sólo morigeraron o corrigieron algunos de sus aspectos, a fin de mitigar los excesos del absolutismo monárquico. Mencionamos brevemente algunos de estos aportes.

El origen del poder: La concepción del poder como originario de la comunidad no era totalmente nueva para la época de Hobbes, ya que había sido de alguna manera adelantada por algunos pensadores de la Iglesia. El pensamiento liberal formaliza esta concepción a través de las teorías contractualistas de Hobbes, Locke y Rousseau, luego completadas técnicamente con la impecable síntesis de la teoría de la representación política de Sieyes. El poder se origina en el pueblo -entonces llamado Nación o comunidad- y es transferido al gobernante por la vía de la teoría de la representación política y del mandato libre.

La extensión del poder: Típicamente en el marco de las monarquías absolutas el poder era ilimitado. En el pensamiento liberal, el ejercicio del poder tiene limitaciones muy concretas cuyas fuentes son:

- La división del ejercicio material de las funciones entre Legislativo, Ejecutivo y Judicial en un sistema de contrapesos, equilibrios y balances.

- El respeto a los derechos individuales que son inherentes al ciudadano e implican un límite a todo posible avasallamiento por el Estado.

- La constitución escrita y rígida como carta de garantías de los principios reseñados

- La responsabilidad de los funcionarios por las consecuencias de sus actos

La temporalidad del ejercicio del poder: En el marco del poder absoluto de los monarcas éste se extendía naturalmente a lo largo de toda la vida. A partir de la emergencia del modelo representativo republicano, la 
periodicidad de las funciones se erige en una característica central del modelo.

La aparición del constitucionalismo liberal acompañó la circunstancia histórica de la llegada de la burguesía al poder. A fines del siglo XIX, con la emergencia de otra nueva clase social, la del proletariado industrial, nuevas necesidades sociales insatisfechas requirieron la aparición de otras ideologías, como los socialismos, el marxismo y la doctrina social de la Iglesia. En ese contexto, el constitucionalismo se enriquece con la cuestión social.

Es con el marxismo que el poder comienza a visualizarse como un fenómeno sistémico o estructural -no como algo meramente interpersonalque se reproduce e internaliza dentro del tejido social o del sistema de relaciones sociales. En el caso concreto de Marx, el autor plantea que el poder deriva de las relaciones sociales de producción, y que su división en económico, político, ideológico, etc., es analítica y no está en la naturaleza de las cosas.

Evidentemente el poder se carga de un aspecto peyorativo que lo acerca a conceptos como el de dominación o hegemonía (en Gramsci). A su vez, como la mayoría de estas teorías eran a su vez programas de acción política, buscaban acceder al poder para producir un cambio en las estructuras sociales. Cabe remarcar que Marx entendía que la burguesía accedía al poder mediante la economía, el proletariado debía hacerlo a través de la política. Esa observación implica un aporte renovador en la ciencia política clásica en lo relacionado a la problemática del poder. La emergencia a lo largo del Siglo XIX de estas nuevas corrientes de pensamiento, muchas de ellas orientadas a la acción, darán lugar a la aparición de las ideologías políticas rígidas y prescriptivas y del denominado "modelo delegativo partidocrático"16, un modelo que hoy se encuentra en una crisis profunda.

\section{Las dudas que plantea el concepto tradicional}

La noción clásica de poder hasta aquí reseñada, entendida como “capacidad para imponer determinadas conductas a otros" nos plantea dudas centrales. ¿Quién tiene realmente ese poder? Si enfrentamos ejemplos clásicos de la literatura nos surgen más interrogantes que respuestas. ¿Tiene realmente poder un padre sobre su hijo, cuando le ordena hacer algo que éste no quiere hacer? $\dot{¿}$ Tiene ese poder la maestra cuando intenta guardar la disciplina en el grado o hacer que sus alumnos estudien un tema o hagan una tarea? $\dot{¿}$ Tiene ese poder el Presidente cuando desea que los precios no aumenten? ¿Tiene ese poder el gobernante que desea impulsar un proyecto o una determinada decisión que encuentran resistencia? ¿Tiene ese poder

\footnotetext{
${ }^{16}$ Montbrun, Alberto; "La crisis de las ideologías y la reinvención de lo político en el Siglo XXI”; Revista 19, San Juan, Marzo de 2006.
} 
el jefe policial que ordena a sus subordinados el cumplimiento de determinadas tareas? Son infinitos los casos en los que advertimos que ese poder no existe realmente y que circunstancias de todo tipo condicionan la real eficacia de las decisiones autoritativas que se desean imponer.

\section{La perspectiva desde el paradigma científico autoorganizativo}

Durante el siglo XX, el paradigma científico positivista, reduccionista y mecanicista de cuño cartesiano - newtoniano tuvo significativas revisiones que cambiaron nuestra percepción de la realidad e implicaron una transformación profunda de los modelos mentales con los que analizamos y operamos los sistemas vivos ${ }^{17}$. La emergencia de un nuevo paradigma científico, evolucionista, autoorganizativo y holístico, permite reexaminar muchas de las suposiciones básicas sobre las que asentamos nuestra comprensión del funcionamiento de la sociedad, revisando críticamente viejos mitos y consolidadas presunciones.

El nuevo enfoque parte de la premisa de que para desentrañar una realidad compleja no es correcto abordarla tratando de explicar o entender sus partes individualmente o aisladas unas de otras. Por el contrario, postula que el funcionamiento de un sistema complejo sólo puede describirse entendiendo sinérgicamente las interacciones, relaciones e intercambios entre sus componentes y del sistema con el entorno en el que opera. Aún más: para el moderno enfoque sistémico - cibernético un sistema no está compuesto de partes sino de relaciones, procesos e interacciones.

En el presente trabajo destacamos algunas características de los denominados sistemas adaptativos complejos (SAC) y de teoría de redes a fin de indagar qué novedad pueden aportar a una teoría científica del poder que permita clarificar las imprecisiones y carencias de las doctrinas clásicas, si es que una tal teoría del poder tiene sentido en la realidad actual.

En general podemos definir un SAC como uno que presenta las siguientes características:

- Indefinido número de elementos y componentes

- Relaciones no lineales y asimétricas entre esos elementos

\footnotetext{
${ }^{17}$ Puede verse Capra, Fritjof; La trama de la vida; Anagrama, Madrid, 1996; Nemeth Baumgartner, Antonia; Macrometanoia. Un nuevo orden. Una nueva civilización. El cambio de paradigma científico en las ciencias jurídicas, políticas y económicas; Ed. Sudamericana, Santiago de Chile, 1994.. Sobre su impacto en la política sugerimos Montbrun, Alberto; El cambio en la ciencia, el cambio en la política; en "Sociedad vs. Política", Zeta Editores, Mendoza, 2002. También en www.albertomontbrun.com.ar
} 
- Procesos de retroalimentación entre los componentes del sistema

- Conducta que puede aparecer azarosa o desordenada pero que responde a patrones subyacentes

- Conducta impredecible, dentro de umbrales de cierta estabilidad

• Evolución por hiperciclos

- Apertura

- Sensibilidad al entorno

- Patrón de red

Los sistemas de actividad humana, en cualquier ámbito en que se verifiquen, son también sistemas adaptativos complejos, que responden a las características mencionadas. Dentro de ellos, debe considerarse naturalmente los sistemas y procesos relacionados con la actividad política o la actividad educativa.

\section{Características de los SAC que controvierten el concepto tradicional de poder}

Dentro de las características de los SAC hay algunas que necesariamente impactan en la idea de "poder" como imposición racional de voluntad. Por ejemplo, la no linealidad. En los SAC cualquier proceso de actividad humana está sometido a un número significativo -cuando no infinitode variables que interactúan de manera asimétrica y dispar, entre sí y con el entorno, operando efectos de tipo no lineal. Dada esta interacción de múltiples y diversos factores la conducta de estos sistemas está sometida a una evolución de tipo no lineal. La no linealidad plantea también la existencia de diferentes tipos de efectos a partir de una misma causa en el análisis de cualquier interacción social. Una ordenanza municipal, por ejemplo, que es para la doctrina clásica un hecho de poder, genera efectos absolutamente dispares en el colectivo sobre el cual está destinada a operar, pudiendo los mismos variar en un rango que va desde el acatamiento pacífico hasta la más grosera violación o la resistencia militante. Aún más, el derecho conoce la situación de ordenanzas y leyes que sin ser expresamente derogadas son olvidadas y dejan de cumplirse.

En forma similar a la no linealidad opera la impredictibilidad, ya que considerando la compleja interdependencia de los elementos e interacciones que operan en un SAC, su exacto estado en cada momento del futuro es desconocido e impredecible. La ciencia puede ponderar o considerar un infinito número de futuros posibles, pero un solo estado futuro de un sistema es impredecible. En materia de "poder" la situación es aún más compleja porque, siendo los seres humanos sistemas autopoiéticos, su 
respuesta frente al input de una orden o un gesto de poder no dependerá de dicha orden o gesto sino de la propia y particular forma en que el sistema procesa el input desde su particular, única e irrepetible configuración sistémica y clausura organizacional.

Los componentes individuales de un sistema se influencian recíprocamente y se relacionan unos con otros, condicionando mutuamente sus conductas y reacciones. Interdependencia e interacción tienen que ver con las modalidades de acoplamiento estructural que desarrollan los sistemas vivos entre sí y con el entorno, en función de mantener su homeostasis. En las campañas electorales los partidos fijan sus propias estrategias, pero las mismas dependerán fuertemente de los inputs provenientes de las estrategias de los otros partidos y del propio entorno.

Humberto Maturana expresa al respecto que "como las propiedades y características de cada ser vivo están determinadas por su estructura, en la medida en que las estructuras de los seres vivos que integran un sistema social cambian, cambian sus propiedades y el sistema social que generan con sus conductas también cambia”18 .

Los sistemas complejos interactúan entre sí a través de un intercambio permanente de flujos de energía, materia e información que los van transformando. Estos procesos activan ciclos o bucles de retroalimentación entre los sistemas -y dentro de ellos entre sus distintos subsistemasque tienen en general la función de mantener la homeostasis compensada con el entorno. Decimos compensada y no "equilibrada” ya que los SAC operan siempre alejados del equilibrio.

Cuando un gobierno enfrenta una creciente protesta social traducida en piquetes y cortes de rutas como la que vivió nuestro país hace pocos años, la falta de respuestas a las demandas de los piqueteros actuaba como un fuerte motor de retroalimentación positiva, al extremo que era diario el corte de rutas y autopistas. Entre 2003 y 2007 el presidente Kirchner decidió responder proactivamente a la presión de estos sectores, dando respuestas materiales y simbólicas que le permitieron compensar homeostáticamente dicha presión, reduciéndola significativamente y logrando incluso, con ciertos sectores, volcarla a su favor. Pero a partir del 2008, otros sectores no contenidos comenzaron estas actividades lo que suscita otra necesidad de respuesta.

Como estructuras disipativas, abiertas a múltiples intercambios con el entorno, pero cerradas desde el punto de vista organizacional, los SAC pueden volverse sistemas altamente desorganizados. En algún punto, el sistema se reorganiza a sí mismo en una nueva estructura, que tendrá generalmente un mayor nivel de complejidad. Esta nueva estructura aparece como

\footnotetext{
${ }^{18}$ Maturana, Humberto; Transformación en la Convivencia; Ed. Dolmen, Chile, 1999.p. 27
} 
una consecuencia de la conducta emergente de todo el sistema. Este principio es el llamado de autoorganización. La idea de autoorganización de los sistemas adaptativos complejos pone en fuerte entredicho las nociones tradicionales de "control externo" y "regulación jerárquica de procesos” como así también cualquier posible referencia al "equilibrio" que es un término que bien podría ser descartado de las ciencias sociales y reemplazado por la expresión "compensado homeostáticamente”. Estando siempre el concepto tradicional de poder referido a un input que el que obedece recibe del que manda, está claro para nosotros que el principio de autoorganización también lo controvierte ya que, como hemos señalado, el sistema se autoorganiza a partir de su propia configuración estructural.

Los procesos de autoorganización se desarrollan en todos los SAC. Por ejemplo, cuando comienza anualmente nuestro curso de Derecho Político, los alumnos son invitados a conformar grupos de trabajo y reciben las consignas de los mismos. Ahora bien, la forma específica que cada grupo adopta para trabajar y cumplir los outputs esperados es propia de la autoorganización de cada uno, que además evoluciona en el tiempo impactando a veces sobre la estructura del grupo. Aunque el profesor les diga cómo tienen que actuar u organizarse, cada uno lo hará conforme a su propia, única e irrepetible configuración sistémica. Por ello, la “orden” del Profesor es tan solo un flujo de energía e información que dispara múltiples estados posibles en cada uno de los sistemas que lo reciben.

En tanto sistemas autoorganizativos, los SAC satisfacen las características de apertura al entorno; operación alejada del equilibrio; relaciones no lineales entre los componentes y fluctuaciones en el sistema y variaciones del entorno que disparan cambios de estado ${ }^{19}$. Piénsese tan sólo en el funcionamiento de los partidos políticos, o de los bloques legislativos en las cámaras y se advertirá que siempre los modos concretos de operación son diferentes en cada momento histórico porque atienden a la particular configuración de cada sistema, más allá de que existen patrones recurrentes similares.

Un sistema vivo mantiene una historia de perturbaciones con el entorno, que disparan dinámicamente sus propios estados posibles que operan a la manera de una respuesta que permite mantener al sistema acoplado estructuralmente con ese entorno, manteniendo su homeostasis, este es el fenómeno denominado acoplamiento estructural.

Pero el sistema no solo "responde" reactivamente a los inputs del entorno -un elemento clave en la primera sistémica eastoniana- sino que además genera o produce modificaciones del entorno a partir de las cuales puede lidiar más eficazmente con él. Es decir, reacciona y al mismo tiempo acciona, generando el proceso de coevolución.

${ }^{19}$ Francois, Charles; International Encyclopedia of Systems and Cibernetics; KG Saur, Munich, 1997. p. 309 
Los SAC conservan su estructura y su organización en tanto y en cuanto mantienen su capacidad homeostática para lidiar con un entorno complejo. Las amenazas que enfrentan desde el entorno son, precisamente, las que pueden afectar al sistema como tal y los factores que los mantienen compensados tienen que ver con la capacidad operativa necesaria para mantener su identidad. El concepto de acoplamiento estructural es útil para comprender algunos aspectos relacionados con el concepto tradicional de poder. Si un sistema autopoiético recibe el input de una orden y tiene la percepción de la conveniencia o legitimidad de su cumplimiento seguramente la cumplirá; de lo contrario, posiblemente no lo haga. De nuevo, la cuestión del "poder" dependerá de la forma concreta en que dicho sistema actúe en un momento determinado.

\section{Aportes de la teoría de redes que afectan el concepto tradicional de poder}

La teoría de redes ha venido ocupando en los últimos años un espacio creciente en la ciencia política, relacionada principalmente con la crisis y transformación de las viejas organizaciones jerárquicas y verticales, propias del mecanicismo en nuevas ingenierías del tipo de las "organizaciones inteligentes" u otras.

Los trabajos de investigadores como Stanley Milgram ${ }^{20}$ Duncan Watts ${ }^{21}$, Albert - Lazlo Barabasi ${ }^{22}$, Mark Granoveter ${ }^{23}$ y otros aún más tempranos como el del premio Nobel de Economía, Thomas Schelling ${ }^{24}$, han permitido sistematizar algunos principios del funcionamiento de las redes, que implican también nuevas visiones para la revisión del concepto de poder.

Las redes son heterogeneidades organizadas en función de un fin colectivo común. En cuanto tales, consisten en un conjunto de elementos (nodos) que se conectan a otros elementos por medio de líneas o conexiones. Son particularmente heterogéneas porque si los nodos fueran homogéneos poco circularía por ellas. Es importante destacar que las redes no existen por sí mismas sino que se definen por lo que hacen una vez que son recortadas por la mirada del observador.

Las redes siempre tienen las características de los sistemas adaptativos complejos y configuran un intercambio infinito de flujos de materia, energía e información que operan procesos de transformación en-

\footnotetext{
${ }^{20}$ Publicó originariamente la tesis de los seis grados de separación en The Small World Problem; Rev. Psicology Today - 2; 1967

${ }^{21}$ Small Worlds; Princeton University Press, Princeton, 1999

${ }^{22}$ Linked; Penguin Group, Mass. 2003

${ }^{23}$ Threshold Models of Collective Behavior; American Journal of Sociology, Vol. 83, 1978

${ }^{24}$ Micromotives and Macrobehavior; W. W. Norton, Ney York, 1978.
} 
tre los nodos que integran la red, entre las sub redes de la red y entre la red y el entorno.

La red se ejerce por medio de las conexiones. Un punto no conectado se sitúa fuera del sistema. Sin conexión no hay redes. Por ello, la medida de la red es el número de conexiones, no de puntos o nodos que la integran.

Advirtamos desde ya que muchas de las características de las redes sociales que mencionaremos brevemente a continuación, contribuyen a poner en duda la noción tradicional de poder como capacidad de determinar la conducta de otras personas. Esto, debido fundamentalmente a que las personas, como nodos de la red social, están permanentemente sometidas a un infinito flujo de insumos de materia, energía e información provenientes de los más dispares subsistemas sociales y por lo tanto la conducta individual de cada persona, en cada momento, resulta algo absolutamente impredecible, más allá de patrones culturales de tipo general.

a. Relaciones no lineales. Un mensaje o una conexión puede extenderse en muchas direcciones ya que no existe un camino único de un punto a otro, pudiendo incluso volver al origen en forma de retroalimentación. Al mismo tiempo, un mismo flujo de información afecta de manera muy diversa a cada uno de los nodos a los que llega, generándose a partir de allí las más diversas retroalimentaciones. La misma disposición legal -en tanto flujo de información- es receptada de manera muy distinta por los ciudadanos y, a su vez, esa diversa recepción genera también distintos intercambios con los decisores.

b. Auto regulación. Los elementos del sistema se regulan y organizan a sí mismos en base a su experiencia, errores y aciertos y sobretodo en base al propósito común que les une. La coordinación es una co-producción de todos. La organización es siempre un proceso y no un estado final. Las redes, como todo sistema vivo, aprenden y evolucionan desde la experiencia. La lógica operativa de una red no surge de ninguna imposición externa sino de la forma específica, única e irrepetible en que cada red absorbe y procesa los inputs del entorno.

c. No jerárquica. No hay niveles verticales, solamente redes animadas dentro de redes y reflejadas en su horizontalidad. Existe un orden en una dinámica de auto ajuste recíproco, sin un control central de poder. Se establecen relaciones de paridad, relaciones de equilibrio y equidad. Ciertamente dentro de la realidad política hay algunos nodos que por su especial situación o posición disponen de una mayor capacidad para la emisión de energía, materia o información (vg. un Intendente o un Presidente) pero esta es siempre una situación fáctica a analizar en cada caso concreto ya que responde a la lógica de las interacciones de la red y no a disposiciones legales o autoritativas.

d. Abierta. Las redes son sistemas abiertos, vivos, que suman nuevos elementos constantemente. Como todo sistema interactivo de conduc- 
tas humanas las redes suman nuevos actores y restan a los que por una razón u otra salen del sistema. Pero la apertura no solo involucra flujos de personas sino también de información, materia y energía desde el entorno. No hay en la realidad sistemas cerrados.

Sin embargo, sí es factible advertir sistemas humanos que por sus especiales características podrían ser vistos como "predominantemente cerrados” si atendemos a las condiciones de acceso a los mismos. Así, la partidocracia sólo permite el ingreso al sistema de aquellos nodos cuyos modelos mentales y conductas no ponen en peligro la estabilidad del sistema. Las universidades sólo permiten la promoción de cuadros académicos que en general no plantean rupturas con el status quo. Algunos clubes o grupos humanos tienen sistemas particularmente rígidos de acceso.

e. Crecimiento y plasticidad. Derivan de la apertura de las redes. Conexiones producen nuevas conexiones. Aprendizajes y experiencias generan nuevas estrategias y métodos de coevolución. Las redes en principio están en constante crecimiento. No obstante, pueden verificarse procesos de incremento de la entropía por falta de incorporación de insumos energéticos dinamizadores y en ese caso la red puede entrar en un proceso de ralentización y decrecimiento.

Algunos partidos políticos, por ejemplo, pierden su capacidad de generar nuevos ingresos en el entorno social y comienzan a languidecer o incluso extinguirse. En ocasiones, recurren a estrategias parasitarias -como por ejemplo determinados tipos de alianzas- que les permitan seguir esperando apoyos o votos.

f. Sin un centro. Dependiendo del punto de vista, del punto de partida, cada punto conectado puede ser un centro. No hay una centralidad sino muchos centros. Aún si aceptáramos la idea tradicional de poder, veríamos que en la red no está concentrado sino distribuido, diluido. Cada elemento es un colaborador del todo, parte del sistema que es de todos, donde hay multiliderazgo. Esto podemos visualizarlo gráficamente cuando superponemos el organigrama formal de un organismo - por ejemplo, un municipio- con la red política y social real en la que ese municipio opera y de la forma en que se adoptan e implementan las decisiones. Podremos ver, por ejemplo, que si bien todas las Direcciones municipales tienen el mismo nivel jerárquico, algunas configuran nodos infinitamente más conectados que otras. $\mathrm{O}$ que el Intendente, que aparece en el máximo nivel decisional, no siempre es el nodo más crítico de la red porque una autoridad partidaria o una autoridad política de afuera del municipio pueden tener más capacidad de generación de flujos decisionales, es decir pueden ser hubs o conectores más relevantes.

g. Redundancia. La característica de las redes, que no se verifica en general en las organizaciones humanas, es que entre dos nodos existen múltiples caminos alternativos. Eso se relaciona a su vez con la robustez de la red ya que la desaparición de un nodo en principio no afectaría la conectividad del sistema por la existencia de múltiples vías alternativas. 
En las organizaciones de vinculación piramidal, las personas se estructuran en distintos niveles de jerarquía que se van superponiendo apoyados sobre una base. Para llegar al vértice -donde se encuentra el líder o jefe único- es necesario escalar cada nivel. Las redes, en cambio, son alternativas al modelo piramidal ya que sus integrantes se articulan de manera horizontal. En las redes, hay una voluntad colectiva de lograr un objetivo compartido. Las redes no son en sí mismas absolutamente nada. Las redes "existen para", como un medio que posibilita buscar respuestas alternativas. Como en todas las relaciones humanas, los vínculos en la red se construyen, se alimentan, se sostienen y también se deterioran o se destruyen.

Es interesante desatacar que, en cuanto sistemas vivos, los SAC de tipo disfuncional -como la burocracia, la partidocracia, el clientelismo, la delincuencia o la corrupción- tienen un patrón de red. Esto implica que tienen un subsistema de mantenimiento o control "distribuido" (en lo personal, lo considero un verdadero sistema inmunológico) y por lo mismo, extraordinariamente eficaz a la hora de enfrentar perturbaciones de entorno. Ejemplos recientes muestran esta interesante contradicción. El ejército de Estados Unidos, tal vez el más poderoso de la tierra, no puede lidiar contra la resistencia iraquí porque esta opera con un patrón eminentemente heterárquico y por lo mismo autoorganizativo. Algo similar sucedió en la guerra de Viet Nam. El fracaso del ejército israelí en su incursión contra Hezbolah en el sur del Líbano en 2006 se inscribe en la misma lógica, al confrontar a una organización mecánica, rígida y altamente profesionalizada con una organización flexible y "viva” con permanente cambio y adaptación al medio y con un solapamiento intersticial con ese medio.

La equiparación del concepto de poder a alguna variante de la fuerza es siempre una tentación para el analista. Sin embargo, también platea inconvenientes. Cuando nosotros movemos un objeto este se mueve por la energía que nosotros le estamos aplicando, pero cuando damos una orden a una persona ésta la responderá, cualquiera sea la forma en que lo haga, desde su propia energía y desde su propia configuración autopoiética. Por ello, cuando enfrentamos sistemas autopoiéticos, debemos comprender que la determinación de la conducta no surge del input sino de la forma específica y concreta en que cada sistema autopoiético procesa y metaboliza dicho input y opera su respuesta.

Tampoco podemos concebir las relaciones de poder como relaciones que se dan entre dos sujetos, sin considerar de manera crítica el entorno en el cual esa relación opera y la forma en que dicho entorno condiciona a los participantes del proceso.

Como señala Vivianne Amaral ${ }^{25}$, cualquier parte nodo de la red puede poner información en circulación. La cosa es aún más dramática: en la ac-

\footnotetext{
${ }^{25}$ Amaral, Vivianne; Redes: Uma nuova forma de atuar; (mimeo) (se encuentra en la biblioteca del Programa en el eRITS y en el Portal ABDL).
} 
tualidad cualquier nodo de la red puede colapsar la red. Piénsese tan solo en Setiembre 11, donde un ínfimo número de personas con un ínfimo costo económico produjo una hecatombe mundial de consecuencias devastadoras. También resulta gráficamente ejemplificador como “efecto mariposa”.

En realidad, las organizaciones “verticales” también tienen un patrón de red en su dinámica y funcionamiento -porque son sistemas vivospero se encuentran en contradicción permanente entre lo que la estructura pretende y lo que la red efectivamente opera. Esto se puede ver muy bien en los casos en que analizamos una estructura vertical donde muchas veces quien está en lo más alto de la pirámide carece de una efectiva capacidad para movilizar procesos e implementar efectivamente decisiones.

Otro caso interesante de contradicción entre sistemas mecánicos en jerarquía piramidal y sistemas vivos en red se produce por ejemplo en Brasil cuando hace más de una década el narcotráfico comienza a reclutar jóvenes sin antecedentes penales y facilita su ingreso como agentes del sistema penitenciario. Cuando años después el gobierno desea imponer ciertas decisiones en ese sistema se encuentra sin capacidad real para operarlas porque los agentes penitenciarios acatan las ordenes de sus "superiores jerárquicos” que son los propios jefes narcos allí alojados. De nuevo, la contradicción entre sistemas orgánicos y sistemas mecánicos se resuelve a favor de los primeros.

\section{Conclusiones provisorias}

De múltiples análisis realizados en clases y seminarios hemos detectado una serie de conceptos asociados a la idea de "poder” que aparecen enumerados en la primera columna del Cuadro 1.

\section{Cuadro 1: "Poder" y conceptos relacionados en ambos paradigmas}

\begin{tabular}{|c|c|}
\hline CONCEPTO TRADICIONAL & $\begin{array}{l}\text { NUEVOS ENFOQUES } \\
\text { PARADIGMATICOS }\end{array}$ \\
\hline Fuerza & Ejemplo \\
\hline Cosacción & Estimulo \\
\hline Coerción & Promoción \\
\hline Imposicion & Facilitación \\
\hline Autoridad & Influencia \\
\hline Amenaca & Negociación \\
\hline Sanción & Transacción \\
\hline Manipulación & Persuasión \\
\hline $\begin{array}{l}\text { Emoción prodominante: } \\
\text { Temor } \\
\end{array}$ & $\begin{array}{l}\text { Emocion predominante: Empatia / } \\
\text { Amor }\end{array}$ \\
\hline Tipo de participación: Movalizada & Tipo de participacón: Autónoma \\
\hline Estructura: la piramide & Estructura: la red \\
\hline
\end{tabular}


En los mismos se advierte siempre la idea subyacente o explícita de coacción o imposición. Es interesante señalar que cuando se indaga sobre las emociones subyacentes, el temor aparece siempre como la principal. En la red, por el contrario, predominan los conceptos enumerados en la columna de la derecha, que están relacionados con procesos interactivos propios de la relación entre sistemas autopoiéticos. Allí, la empatía o el amor aparecen como las emociones predominantes. Respecto al tipo de participación, distinguimos en términos de Huntington ${ }^{26}$ una participación "movilizada" es decir inducida por terceros y una autónoma a partir de la propia decisión de la persona que participa.

A nosotros nos parece que una concepción del poder como "la energía distribuida en la red” y su comprensión en términos cuánticos, flexibles y situacionales permite comprender mejor cómo en determinadas situaciones las mismas personas actúan de manera distinta o por qué, por ejemplo, más allá de lo que diga un organigrama o un manual de funciones, las personas operarán siempre de manera básicamente impredecible.

Los nodos de la red procesan y emergen energía, materia e información a partir de su propia configuración autopoiética. Al mismo tiempo “determinan” la conducta de otros sistemas sí y solo sí la configuración autopoiética de los mismos permite esa posibilidad.

En todo caso, podríamos pensar el poder como la energía vital que todo sistema utiliza, ya sea para generar estrategias de reducción de variedad del entorno, tratando de inducir o determinar conductas; o bien para incorporar variedad sistémica a través de procesos activos de aprendizaje.

La idea central de Toffler en "La Tercera Ola” y “El Cambio en el Poder" es el paso de una sociedad masificada - la industrial - a otra de tipo desmasificada y la importancia de la información como factor de poder en detrimento de la fuerza y el dinero. El concepto más elemental de poder con el cuál trabajan los autores clásicos parece tener vigencia en el contexto de la sociedad industrial. En este tipo de sociedad masificada, al decir de Toffler, obviamente la variedad del entorno para el sistema político es menor. Con el nacimiento de una nueva sociedad, plural, desmasificada y, principalmente, informada, la variedad a la cual deben hacer frente los sistemas que formalmente toman las decisiones autoritativas de valor, ha aumentado.

Algunos aportes al debate sugieren que no es el concepto de poder lo que estamos desafiando, sino su lógica de distribución, reproducción y utilización. Si entendemos al poder como un flujo energético (genera estímulos) consiente o inconsciente, el cual se manifiesta de diversas formas (concretas o simbólicas), que opera por múltiples medios y de manera constante sobre el dinamismo de las relaciones sociales; el principio de acción

\footnotetext{
${ }^{26}$ Huntington, Samuel; "El sobrio significado de la democracia". Revista de Estudios Públicos N³3. Santiago, 1989.
} 
de la ecuación clásica de poder (acción y reacción), seguiría existiendo. Es sobre la segunda etapa de la ecuación que la lógica del poder cambia radicalmente con el cambio de paradigma. Quizás el hecho de tener arraigado a nuestras estructuras mentales (o matrices de pensamiento) el funcionamiento de poder como lo describían los positivistas, nos hace que, consiente o inconscientemente, utilicemos esta lógica del poder lineal para lograr efectos en nuestras relaciones sociales. De allí que de alguna manera este concepto siga vigente a la hora de crear análisis social. Evidentemente la complejización del sistema social y su disposición en redes, nos lleva a replantear efectos, retroalimentación, distribución y alcance del poder.

El concepto de poder no demuestra una real utilidad en el estado actual de la ciencia política. Su excesiva vaguedad y ambigüedad lo torna un concepto que no expresa ni trasunta una realidad fáctica concreta y observable. Al contrario, cada "situación de poder" debe ser explicada.

Tal vez debamos advertir que para resolver esta compleja cuestión debemos clarificar paradigmáticamente nuestra percepción de la realidad. La geometría euclidiana inspiró la graficación de la ideología del poder en formas rígidas y perfectas, que hoy se pueden visualizar en un organigrama. En cambio, en la actualidad, el salto cuántico nos lleva a la inmersión en una sociedad red, donde la geometría de los procesos adopta las formas irregulares y difusas de los fractales.

Como síntesis de esta nueva visión, señala Antonia Nemeth Baumgartner ${ }^{27}$, que: "A través de los aportes de la Ciencias de Complejidad, se presenta la visualización del "Poder” como un hiperfenómeno proveniente de las dinámicas de las interacciones de los componentes de un sistema en busca de objetivos. Se configura este como un "efecto emergente" de la armonización y capacidad negociadora de los subsistemas que concurren en la totalidad de un sistema en su dinámica recursiva. Consecuentemente ha surgido una distinta mentalidad, la que es inconcebible para los pontífices de la mentalidad de la dinámica Newtoniana, que son los que gobiernan las universidades y las Naciones Estado y que conciben y utilizan el poder como una propiedad legitimada por el solo hecho del estatus. El poder es difuso y propiedad de un sistema en su totalidad. Los nodos decisorios, es decir a los lideres o los gobernantes solo les queda la posibilidad de descubrir las alternativas inmersas en el conjunto de posibilidades que la dinámica difusa del sistema les ofrece para cumplir los objetivos en función de decisiones óptimas”.

Pensamos que el cambio de paradigma científico resulta de la mayor importancia para entender el cambio en el poder. Sin embargo, el reacomodamiento de la ciencia a lo largo del siglo XX, a través del salto

\footnotetext{
${ }^{27}$ Nemeth Baumgartner, Antonia; Macrometanoia. Un nuevo orden. Una nueva civilización. El cambio de paradigma científico en las ciencias jurídicas, políticas y económicas; Ed. Sudamericana, Santiago de Chile, 1994. p.230
} 
paradigmático de la complejidad, no ha impactado todavía en nuestras instituciones políticas, que siguen operando sobre la base de las viejas ideas del iluminismo racionalista pero desbordadas en todas partes por la dinámica de una realidad que no se deja controlar ni gobernar por nada ni por nadie.

En su suma, el cambio en el poder, desde la concepción newtoniana, mecánica, lineal, rígida, vertical y posicional hacia una concepción cuántica, difusa, incierta, situacional y en red, no ha sido todavía adecuadamente percibido ni por nuestros líderes ni por nuestros científicos sociales y esto retrasa las necesarias transformaciones que nuestra sociedad está reclamando. 


\section{Bibliografía}

Amaral, Vivianne (s/a), Redes: Uma nuova forma de atuar, (mimeo) Disponible en biblioteca del Programa eRITS y en el Portal ABDL.

Arendt, Hannah (1993), La condición humana, Paidós, Barcelona.

Aron, Raymond (1968), Democracia y totalitarismo, Editorial Seix Barral, Barcelona.

Avila Fuenmayor, Francisco (2005), “Algunas Ideas del Pensamiento Político de Hanna Arendt. Su impacto actual”, en Revista de Cencias Sociales, Vol 11, Maracaibo.

Capra, Fritjof (1996), La trama de la vida; Anagrama, Madrid.

Dahl, Robert (1957), “The Concept of Power”, en Rev. Behavioral Science, Vol. II.

Easton, David (1968), Política Moderna, Ed. Letras, México.

Etzioni, Amitai (1978), La sociedad activa. Una teoría de los procesos sociales y políticos, Aguilar, Madrid.

Granoveter, Mark (1978), "Threshold Models of Collective Behavior”, American Journal of Sociology, Vol. 83, 1978.

Foucault, Michel (1976), La volonté de savoir, Gallimard, París.

Idem (1981), Un diálogo sobre el poder, Alianza Editorial, Madrid.

Idem (1988), “El sujeto y el poder”, en Revista Mexicana de Sociología, No. 3.

Francois, Charles (1997), International Encyclopedia of Systems and Cibernetics, KG Saur, Munich.

Friedrich, Carl (1968), El hombre y el Gobierno: una teoría empírica de la política, Tecnos, Madrid.

Huntington, Samuel (1989), “El sobrio significado de la democracia”, en Revista de Estudios Públicos No33. Santiago.

Labourdette, Sergio (1984), El poder. Hacia una teoría sistemática, Ed. Universidad de Belgrano, Buenos Aires.

Laswell y Kaplan (1950), Power and Society, Yale University Press, New Haven. 
Lazlo Barabasi, Albert (2003), Linked, Penguin Group, Mass.

Maturana, Humberto (1999), Transformación en la Convivencia, Ed. Dolmen, Chile.

Melo, Leopoldo (1979), Compendio de Ciencia Política, Depalma, Buenos Aires.

Milgram, Stanley (1967), “The Small World Problem”, en Rev. Psicology Today, 2; 1967

Montbrun, Alberto (2002), "El cambio en la ciencia, el cambio en la política”, en Sociedad vs. Política, Zeta Editores, Mendoza. Disponible en www.albertomontbrun.com.ar

Idem (2006), "La crisis de las ideologías y la reinvención de lo político en el Siglo XXI”, en Revista 19, San Juan, Marzo de 2006.

Nemeth Baumgartner, Antonia (1994) Macrometanoia. Un nuevo orden. Una nueva civilización. El cambio de paradigma científico en las ciencias jurídicas, políticas y económicas; Ed. Sudamericana, Santiago de Chile.

Oro Tapia, Luis (2003), ¿Qué es la Política?, Ril Editores, Santiago de Chile.

Rivera Garcia, Antonio (2002), “Sobre el concepto político de 'autoridad' en Hanna Arendt” en Rev. Daimon, 26-2002

Schelling, Thomas (1978), Micromotives and Macrobehavior, W. W. Norton, New York.

Schermerhorn, Richard (1968), El poder y la sociedad, Paidós, Buenos Aires.

Spranger, Eduard (1966), Formas de Vida, Ed. Revista de Occidente, Madrid.

Watts, Duncan (1999), Small Worlds, Princeton University Press, Princeton.

Weber, Max (1993), Economía y Sociedad, Ed. FCE, México. 